\title{
Electromodulation of the Magnetoresistance in Diluted Magnetic Semiconductors Based Heterostructures.
}

\author{
M.P.López-Sancho, M.C.Muñoz and L.Brey \\ Instituto de Ciencia de Materiales de Madrid (CSIC), Cantoblanco, 28049 Madrid, Spain.
}

\begin{abstract}
We study the properties of heterostructures formed by two layers of diluted magnetic semiconductor separated by a nonmagnetic semiconductor layer. We find that there is a RKKY-type exchange coupling between the magnetic layers that oscillates between ferromagnetic and antiferromagnetic as a function of the different parameters in the problem. The different transport properties of these phases make that this heterostructure presents strong magnetoresistive effects. The coupling can be also modified by an electric field. We propose that it is possible to alter dramatically the electrical resistance of the heterostructure by applying an electric field. Our results indicate that in a single gated sample the magnetoresistance could be modulated by with an electrical bias voltage.

PACS numbers: 75.50.Pp, 75.70.Cn, 75.70.Pa
\end{abstract}

Recently it has been possible to grow Mn doped GaAs semiconductors with a ferromagnetic paramagnetic transition temperature, $T_{c}$, near 100K [1]. Further experimental and theoretical works indicate the possibility to obtain room temperature ferromagnetism in others diluted Mn-doped III-V semiconductors 20, 3, 4.

Room temperature ferromagnetic semiconductors have a great potentiality to be used in magnetoelectronics and spintronics. Therefore a big effort is been doing in two directions: first the study of the origen of ferromagnetism and the search of high Curie temperature magnetic semiconductors $5,6,6,8,9,107$, and second the design and growth of devices and heterostructures for magnetoelectronics and spintronics 11, 12, 13, 14.

The III-V high $T_{c}$ semiconductors have a concentration, $x$, of Mn ions randomly located. Experimentally, the optimal $\mathrm{Mn}$ concentration for obtaining high $T_{c}$ 's is near $x \sim 0.06 \sqrt{15}$, and the magnetic impurities are rather diluted, hence the name Diluted Magnetic Semiconductors (DMS). At small Mn concentration, each ion substitutes a column-III cation $\left(\mathrm{III}_{1-x} \mathrm{Mn}_{x}\right.$-V), gets a $S=5 / 2$ local moment and gives a hole to the host semiconductor. A large amount of these holes are trapped on antisite ntype deep defects present in the host semiconductor, since these materials are grown at low temperatures 15. The rest of holes, with a concentration $p<<x$, are responsible for the occurrence of ferromagnetic order in DMS. The system is formed by two interacting subsystems: a subsystem of $\mathrm{Mn}$ ions which are so dilute that direct interaction between their magnetic moment is negligible, and a subsystem of carriers. Without interaction between them, both systems should be paramagnetic at any temperature. However, the antiferromagnetic Hund's coupling, $J$, between the carrier and the Mn spins makes the two subsystems to become ferromagnetically ordered and antiferromagnetically coupled. The Mn ions feel a long range ferromagnetic interaction mediated by the itinerant spin polarized carriers 2, 3, 4. The Curie temperature of the DMS's depends on the carrier density of states at the Fermi energy, the Hund's coupling and the density of magnetic impurities.

In this work we study the properties of heterostructures formed by two slabs of thickness $d_{M}$ of Mn-based DMS separated by a nonmagnetic semiconductor layer of thickness $d_{P}$. Analyzing this problem in the framework of the mean field approximation, we investigate the electrical, magnetic and transport properties of the heterostructures as function of $x, p, d_{M}, d_{P}$, Hund's coupling, band offset and external bias voltage.

The main conclusions of this work are the following: i) There is a RKKY-type exchange interaction between the magnetic layers that oscillates in sign as a function of the different parameters in the problem. Positive and negative signs correspond to antiferromagnetic $(\mathrm{AF})$ and ferromagnetic $(\mathrm{F})$ coupling between the magnetic slabs. ii)Assuming that the alloy scattering is much smaller in the paramagnetic semiconductor than in the Mn doped layers, we find that the electrical resistivity, for currents flowing parallel to the interfaces, has rather different values for the $\mathrm{F}$ and $\mathrm{AF}$ coupled heterostructures.

iii) We predict that the electrical resistance can be modulated not only with an external magnetic field, but also by applying and external electric field.

DMS's are described by the following Hamiltonian,

$$
H=H_{h}+J \sum_{I, i} \mathbf{S}_{I} \cdot \mathbf{s}_{i} \delta\left(\mathbf{r}_{i}-\mathbf{R}_{I}\right)+W \sum_{I, i} n_{I} \delta\left(\mathbf{r}_{i}-\mathbf{R}_{I}\right) .
$$

Here $H_{h}$ describes the carriers, it is the sum of the kinetic energy of the holes and the hole-hole interaction energy. For the range of carrier density of interest in DMS's, the carrier-carrier interaction is not relevant and we neglect it. We treat the kinetic energy in the framework of the envelope function approximation. In this approach we describe the hole electronic states of the host semiconductor by a parabolic band. For GaAs, the effective mass, $m^{*}$ is $0.5 m_{e}$ [细. The carriers are confined in the whole heterostructure and their motion is restricted to $-d_{M}-d_{P} / 2<z<d_{M}+d_{P} / 2$. The last two terms in Eq.(11) represent the coupling between the carriers and 


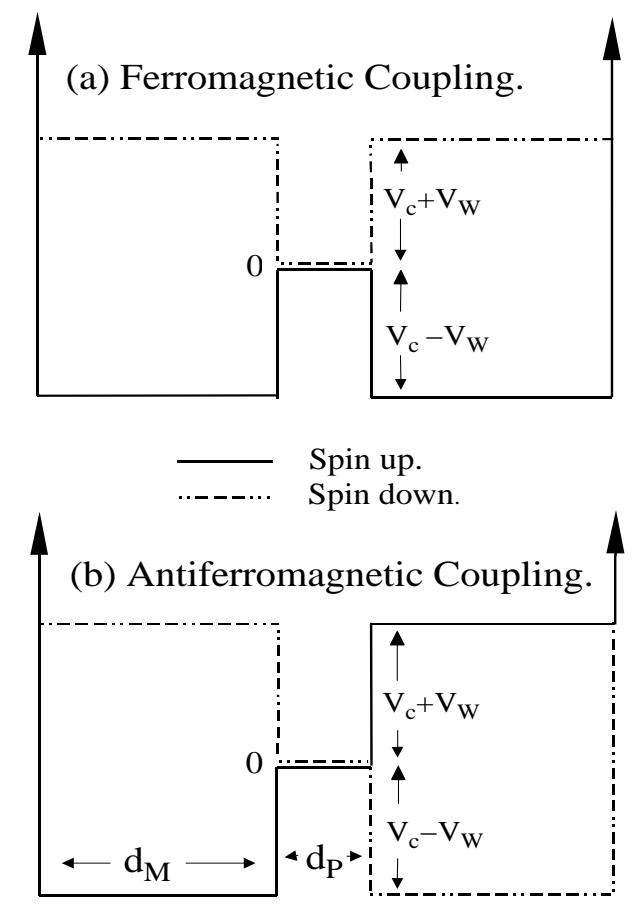

FIG. 1: Potential profiles for both spin carriers directions, in the $\mathrm{F}$ and $\mathrm{AF}$ configurations.

the Mn's. The term proportional to $J$ is the antiferromagnetic exchange interaction between the spin $\mathbf{S}_{I}$ of the $\mathrm{Mn}^{2+}$ ions located at $\mathbf{R}_{I}$ and the spins, $\mathbf{s}_{i}$ of the itinerant carriers. This term is responsible for the long range ferromagnetic interaction between the $\mathrm{Mn}$ core spins. The last term in Eq.(11) is an interaction between the carrier charge, $n_{i}$ and the potential arising from the magnetic dopants. The origin of $W$ is the different electronegativity of $\mathrm{Mn}$ and GaAs atoms, and the screening of the Mn by the carriers. The value of $J$ is typically 0.1$0.15 \mathrm{eVnm}^{3}$ 16, 17, 18, 19]. Since there is not reliable experimental information on the value of $W$, we consider it as a parameter with value $0<W<J$. The direct magnetic interaction between the $\mathrm{Mn}$ ion spins is considerably weaker than the interaction with the carrier spins and therefore we neglect it.

We solve Hamiltonian (1) in the mean field approximation. In this approach, similar to the Jellium model, the local magnetic interaction of the spin carriers with the Mn spins is substituted by the interaction with an effective magnetic field of intensity 20 $S J x / a^{3}$ and directed parallel to the Mn's ion spin polarization. In the same spirit of the Jellium model, the electronegativity difference between the carriers and the $\mathrm{Mn}$ ions is described by an effective potential of interaction $W x / a^{3}$. In these expressions $a^{3}$ is the unit cell volume of the host semiconductor.

Within this approach, in our heterostructure the carriers are free to move in the $x-y$ plane and the one

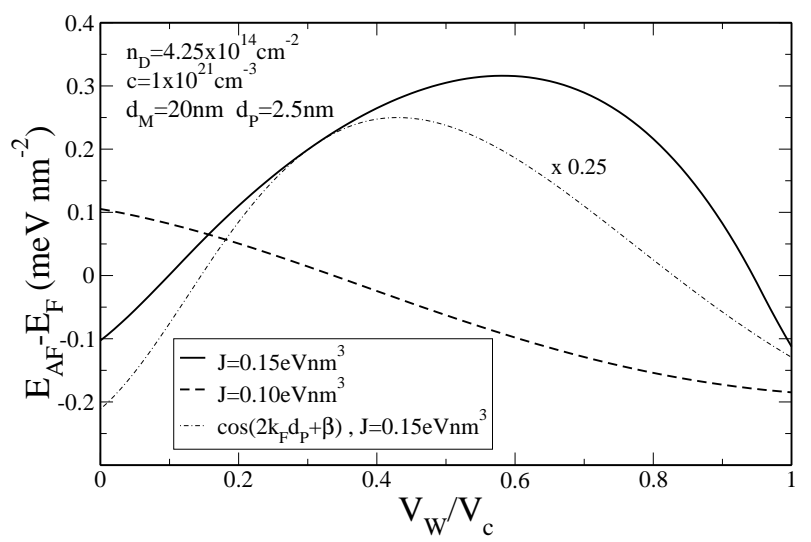

FIG. 2: Energy difference between the AF and the F coupled magnetic layers cases as a function of the bandoffset. We also plot $\cos \left(2 k_{F} d_{P}+\beta\right)$ to show the RKKY origin of the coupling.

particle wave functions and eigenvalues have the form

$$
\Psi_{i, \mathbf{k}_{\perp}, \sigma}^{\alpha}=\frac{e^{i \mathbf{k}_{\perp} \mathbf{r}_{\perp}}}{\sqrt{S}} \Phi_{i, \sigma}^{\alpha}(z), \varepsilon_{i, \mathbf{k}_{\perp}, \sigma}^{\alpha}=\frac{\hbar k_{\perp}^{2}}{2 m^{*}}+\varepsilon_{i, \sigma}^{\alpha} .
$$

Here $S$ is the areal dimension of the sample, $\mathbf{r}_{\perp}$ and $\mathbf{k}_{\perp}$ are the position and the momentum of the carriers in the plane perpendicular to the growth direction, $i$ is a subband index and $\Phi_{i, \sigma}^{\alpha}(z)$ and $\varepsilon_{i, \sigma}^{\alpha}$ are obtained from the one-dimensional Schrodinger equation,

$$
\left(-\frac{\hbar^{2}}{2 m^{*}} \frac{d^{2}}{d z^{2}}+V_{\sigma}^{\alpha}(z)\right) \Phi_{i, \sigma}^{\alpha}(z)=\varepsilon_{i, \sigma}^{\alpha} \Phi_{i, \sigma}^{\alpha}(z),
$$

where $\sigma$ is the carriers spin index, up (+) or down (-), $\alpha$ stands for the solutions with ferromagnetic (F) or antiferromagnetic coupling (AF) between the DMS layers, and the effective potential $V_{\sigma}^{\alpha}(z)$ has the following form, see fig.(11)

$V_{ \pm}^{\alpha}(z)=\left\{\begin{array}{clc}\mp V_{c}+V_{W} & \text { if } & -d_{M}-d_{P} / 2<z<-d_{P} / 2 \\ 0 & \text { if } & -d_{P} / 2<z<+d_{P} / 2 \\ \mp C_{\alpha} V_{c}+V_{W} & \text { if } & d_{P} / 2<z<d_{M}+d_{P} / 2 \\ \infty & & \text { otherwise }\end{array}\right.$

where $C_{\alpha}$ is $+1(-1)$ in the $\mathrm{F}(\mathrm{AF})$ coupling case, $V_{c}=$ $S J x /\left(2 a^{3}\right)$ and $V_{W}=W x / a^{3}$. By summing the energy of the occupied states, we obtain the total energy per unit area of the solutions with ferromagnetic, $E_{F}$, and antiferromagnetic, $E_{A F}$, coupling between the GaMnAs layers.

In fig.(2) we plot the difference $E_{A F}-E_{F}$ as a function of the band-offset, $V_{W}$, for two values of $J$. The parameters of the heterostructure are $d_{M}=20 \mathrm{~nm}$ and $d_{P}=2.5 \mathrm{~nm}$, we consider that the DMS layers have a density of Mn's, $c=1 \times 10^{21} \mathrm{~cm}^{-3}$, and the two-dimensional density of carriers is $n_{D}=4.25 \times 10^{14} \mathrm{~cm}^{-2}$, that roughly corresponds to a three dimensional density of carriers ten times smaller then the density of magnetic impurities. The exchange coupling oscillates as a function of 


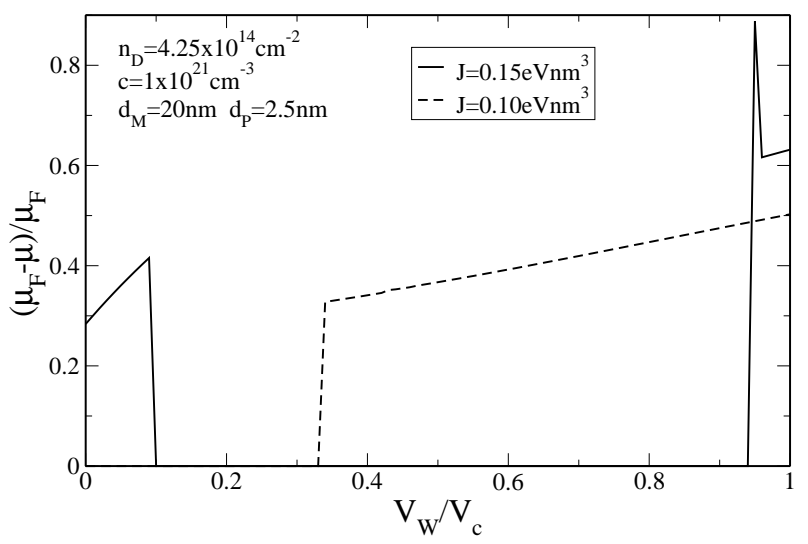

FIG. 3: Absolute value of MR as a function of $V_{W}$.

the band-offset. The coupling is due to a RKKY-like interaction between the DMS layers similar to that occurring in magnetic multilayers 21. The coupling exists because there is charge carriers in the whole heterostructure. In particular in the central region, there is a paramagnetic hole gas which mediates the interaction between the magnetic slabs. To enlighten that, we have plotted the quantity $\cos \left(2 k_{F} d_{P}+\beta\right)$, being $k_{F}$ the Fermi wavevector of the paramagnetic hole gas in the central layer, and $\beta$ an arbitrary phase. From the comparison between the numerical results and the cosine, we conclude that the RKKY model accounts for the existence of the exchange coupling oscillations. The value of the phase $\beta$ has been chosen to make the comparison easier. We have studied the coupling as a function of $W$, but we have also found oscillations in the exchange energy by changing other parameters in the heterostructure which alters the product $2 k_{F} d_{P}$. Similar oscillations in DMS based superlattices have been reported in ref.([14]). Also, indications of interlayer exchange coupling have been observed in GaMnAs/GaAs superlattices 22. For DMS based heterostructures the value of the exchange coupling energy is smaller than in metallic systems. However the magnetic field necessary to overcome the AF coupling is $B \sim \frac{E_{F}-E_{A F}}{g \mu_{B} S c d_{M}} \sim 100-1000$ Gauss, large enough for magnetoresistive applications.

The possibility of changing the layer coupling from AF to $\mathrm{F}$, by applying a magnetic field imply that the heterostructure should present large magnetoresistance (MR) i.e. change of the electrical resistance when applying a magnetic field. The transport parallel to the growth direction should present a MR near 100\%. However the use of this geometry would imply tunneling processes to inject the carriers inside and outside the heterostructure.

Transport parallel to the interfaces also presents large MR. We calculate the conductivity, $\mu$, of the heterostructure assuming that the main source of scattering are the impurities and the antisite defects located in the DMS layers. Assuming point contact interaction between the carriers and the impurities 23], the following expression

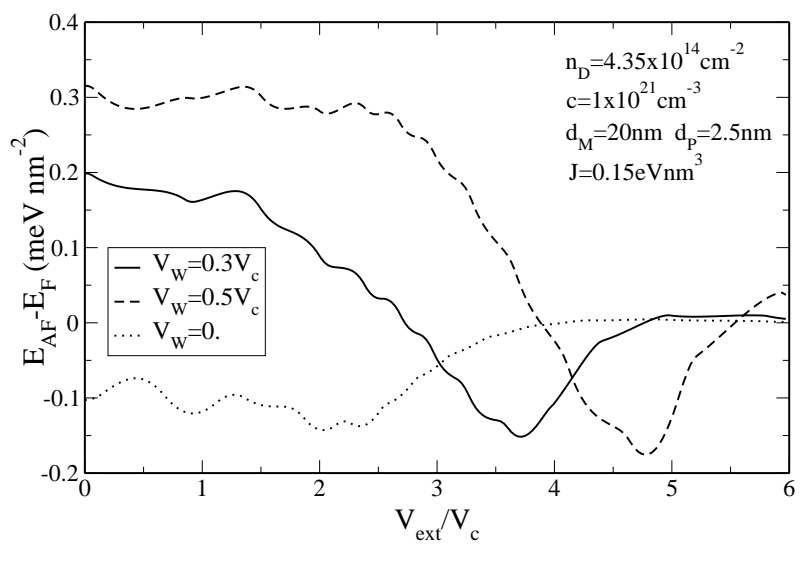

FIG. 4: Exchange energy coupling as a function of $V_{\text {ext }}$.

for $\mu$ is obtained 24

$$
\mu=\frac{e^{2}}{m^{*}} \sum_{i, \sigma} \tau_{i}^{\sigma} \frac{n_{i}^{\sigma}}{n_{D}}
$$

where $n_{i}^{\sigma}$ is the two dimensional density of carriers in the subband $i$ and spin $\sigma$, the sum is over the occupied states and the transport scattering time is given by,

$$
\frac{1}{\tau_{i}^{\sigma}}=C \frac{1}{\sum_{l} A_{i, \sigma, l, \sigma}}
$$

with the sum restricted to the occupied states, $C$ is a constant which depends on the details of the scattering potential and

$$
A_{i, \sigma, l, \sigma}=\int_{\text {imp.region }} d z\left|\Phi_{i, \sigma}(z)\right|^{2}\left|\Phi_{l, \sigma}(z)\right|^{2} .
$$

This integral is restricted to the DMS regions.

We have evaluated the conductivity of the $\mathrm{F}$ and $\mathrm{AF}$ solutions and in fig.(3) it is plotted the absolute value of the MR as a function of the band offset for the cases shown in fig.(2). MR is different from cero, with values bigger than $20 \%$, when the ground state is antiferromagnetic. In the $\mathrm{F}$ case the minority carriers are mainly localized in the central paramagnetic layer, where the scattering is much weaker than in the DMS layers. In the $\mathrm{AF}$ coupling case the carriers, for both spin orientation, located on the central layer have a wavefunction extended on one of the DMS slabs and therefore they suffer a stronger scattering. The minority spin high mobility channels in the $\mathrm{F}$ phase, localized in the central layer, are responsible for the conductivity difference between the $\mathrm{F}$ and the AF phases. The peak that appears near $V_{W} \sim 0.95 V_{C}$ is a quantum effect due to the occupancy of a new subband (see Eq.(6)) in the F phase.

Because semiconductors have lower carrier density than metals, the exchange coupling is sensitive to moderate external electrical bias, $V_{\text {ext }}$ applied from left to right of the heterostructure. In fig.(14) we plot the exchange energy coupling as a function of $V_{e x t}$, for different values 


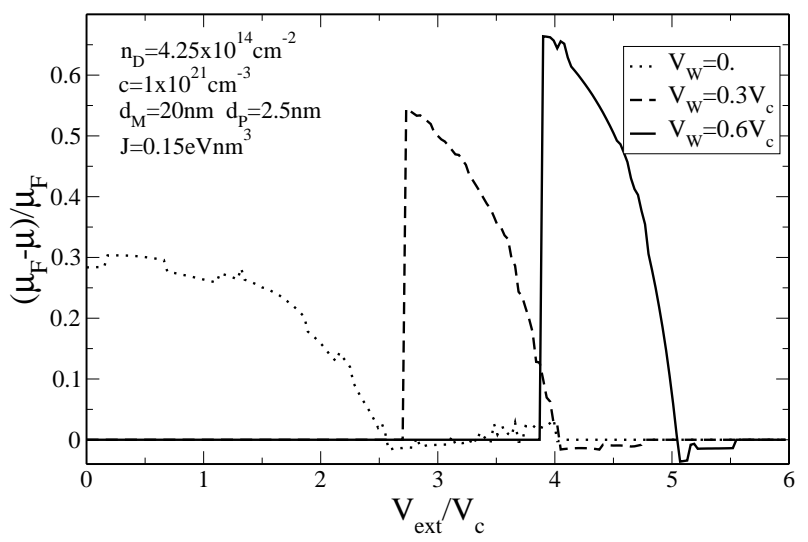

FIG. 5: Absolute value of the MR as a function of $V_{\text {ext }}$.

of $V_{W}$. The application of the bias changes the charge density in the central layers and therefore the coupling oscillates and changes sign. In the cases of $V_{W}=0.3$ and $0.6 V_{C}$ the bias changes the coupling from $\mathrm{F}$ to $\mathrm{AF}$. At large enough bias one of the DMS layers becomes almost depopulated and the two magnetic slabs become decoupled. There is also an superimposed fine structure related to the depopulation of the subbands originated by $V_{\text {ext }}$.

In fig.(可) we plot the absolute value of the MR as a function of $V_{\text {ext }}$. Because the bias changes the coupling from $\mathrm{F}$ to $\mathrm{AF}$, or viceversa, there is a big change in the $\mathrm{MR}$ as a function of $V_{\text {ext }}$. As for the energy coupling there are small oscillations, related with the subbands occupation, superimposed on the mean structure. The main point of the results presented in fig.(5) is the large change in the resistance of the heterostructure when a bias is applied. For the parameter values corresponding to DMS, the magnitude of the electric field necessary to electromodulate the $\mathrm{MR}$ is $\sim 100 \mathrm{KV} / \mathrm{cm}$ that is an experimentally reasonable value.

The results shown in figures (41) and (5) are not only interesting for possible applications in magnetoresistive devices, but also for the basic study of quantum phase transitions. It could be possible to see a quantum phase transition between the $\mathrm{F}$ and the $\mathrm{AF}$ phases in a single gated sample by varying the bias voltage.

In conclusion we have studied heterostructures formed by two layers of DMS separated by a nonmagnetic semiconductor. We find that there is a RKKY-like interaction between the layers that oscillates in sign with the parameters of the problem, and with an applied electrical field. The ferromagnetic and antiferromagnetic coupled heterostructures have rather different resistances and present large magnetoresistive effects. We predict that, for a fixed heterostructure, an external bias changes the coupling from ferromagnetic to antiferromagnetic, producing a big variation on the electrical resistance and magnetoresistance. Therefore the resistance can be modulated, not just with an external magnetic field, but also by applying and external bias voltage.

Financial support is acknowledged from Grants No PB96-0085, BFM2000-1330, BFM2000-1107 (MEC, Spain) and Fundación Ramón Areces.
[1] H. Ohno and F. Matsukura, Solid State Commun. 117, 179 (2001).

[2] T. Dietl, H. Ohno, and F. Matsukura, Phys. Rev. B 63, 195205 (2001).

[3] S. Sonoda, S. Shimizu, T. Sasaki, Y.Yamamoto, and H.Hori, cond-mat/0108159.

[4] J. Konig, J. Schliemann, T. Jungwirth, and A. H. MacDonald, cond-mat/0111314.

[5] T. Dietl, H. Ohno, F. Matsukura, J. Cibert, and D. Ferrand, Science 287, 1019 (2000).

[6] T. Jungwirth, J. Konig, J. Sinova, J. Kucera, and A. H. MacDonald, cond-mat/0201157.

[7] M. Berciu and R. N. Bhatt, Phys. Rev. Lett. 87, 107203 (2000).

[8] A. Chattopadhyay, S. DasSarma, and A. J. Millis, Phys. Rev. Lett. 87, 227202 (2001).

[9] M.J.Calderón, G.Gómez-Santos, and L.Brey, condmat/0203404, Phys.Rev.B in press.

[10] G.Alvarez, M. Mayr, and E.Dagotto, cond-mat/0205197.

[11] L. L. da Silva, M. A. Boselli, I. C. da Cunha Lima, X. F. Wang, and A. Ghazali, Appl. Phys. Lett. 79, 3305 (2001).

[12] J. Fernández-Rossier and L. J. Sham, Phys. Rev. B 64, 235323 (2001).

[13] L. Brey and F. Guinea, Phys. Rev. Lett. 85, 2384 (2000).

[14] T. Jungwirth, W. A. Atkinson, B. H. Lee, and A. H. MacDonald, Phys. Rev. B 59, 9818 (1999).

[15] S.J.Potashnik, K.C.Ku, R.Mahendiran, S.H.Chun,
R.F.Wang, N.Samarth, and P.Schiffer, condmat/0204250.

[16] F. Matsukura, H. Ohno, A. Shen, and Y. Sugawara, Phys. Rev. B 57, R2037 (1998).

[17] H. Ohno, Science 281, 951 (1998).

[18] T. Omiya, F. Matsukura, T. Dietl, Y. Ohno, T. Sakon, M. Motokawa, and H. Ohno, Physica E 7, 976 (2000).

[19] J. Okabayashi, A. Kimura, O. Rader, T. Mizokawa, A. Fujimori, T. Hayashi, and M. Tanaka, Phys.Rev.B 58, R4211 (1998).

[20] In the interaction between the carrier spins and the efective magnetic field, created by the Mn's we take $g \mu_{B}=1$.

[21] U. Hartmann, Magnetic Multilayers and Giant Magnetoresistance. (Springer-Verlag, Berlin, 2000).

[22] R.Mathieu, P.Svdedlindh, J.Sadowski, K.Swiatek, M.Karlsteen, J.Kanski, and L.Ilver, cond-mat/0206380.

[23] We only consider the scattering of the carriers by the electrostatic part of the Mn's impurity potential. At low temperatures spin-flip scattering is considerably weaker. See for example ref(25).

[24] G.Bastard, Wave Mechanics Applied to Semiconductor Heterostructures. (Les Editions de Physique, Les Ulis, 1988).

[25] M.J.Calderón and L.Brey, Phys. Rev. B 64, 140403 (2001). 